\section{Is the Polymyxin B Resistance Among Multidrug-Resistant Enterobacteriaceae (Except for the Carbapenemase-producing Ones) a Myth or a Matter?}

To the Editor-In recent years, polymyxins have been reintroduced into the arsenal of antimicrobial therapy because they are one of the few agents clinically available to treat infections caused by carbapenem-resistant gram-negative bacteria. ${ }^{1}$ Although their clinical usefulness has been put into practice in most treatment approaches, including many empirical therapies, ever-greater resistance has been observed in regions where polymyxins have become more heavily prescribed. $^{2}$

Polymyxins have been widely applied to treat infections related to non-fermentative gram-negative bacteria such as Pseudomonas aeruginosa and Acinetobacter spp., and the rapid emergence of resistance to this class of drug among carbapenem-resistant Enterobacteriaceae (CRE), mostly those Klebsiella pneumoniae carbapenemase (KPC) producers, is notable. ${ }^{3,4}$

While attention is focused on the widespread resistance to polymyxins among KPC producers, with important repercussions for their usefulness, little is known about the polymyxin resistance rates among multidrug resistant (MDR) Enterobacteriaceae with in vitro carbapenem susceptibility. Would selective pressure via the use of polymyxins also act on this type of threat as much as it has on the KPC-producing ones?

To answer this question, a retrospective survey was conducted to assess the prevalence of polymyxin B (PMB) resistance among MDR Enterobacteriaceae. MDR was defined as nonsusceptibility to at least 1 agent in 3 or more antimicrobial categories, including isolates not susceptible to at least 1 carbapenem agent (eg, ertapenem, meropenem, or imipenem), except KPC-producers and those intrinsically resistant to polymyxins. These isolates were recovered from inpatients between January 1 and September 10, 2016, at a tertiary hospital in Porto Alegre, Southern Brazil.

Identification of bacterial species as well as antimicrobial susceptibility testing were initially performed using an automated broth microdilution system (MicroScan; Beckman Coulter, Brea, CA, USA). Polymyxin B minimum inhibitory concentration (MIC) was confirmed using Etest (AB Biodisk, Solna, Sweden). To attribute the resistance mechanism for the enterobacterial species, a synergistic test was applied using phenyl-boronic acid to detect KPC (isolates not included in the study). Enzymatic inhibition testing with clavulanic acid and cloxacillin was used to detect extended spectrum $\beta$-lactamases (ESBLs) and AmpC enzymes, in that order, as previously described. ${ }^{5}$

During the study period, 67 enterobacterial isolates presenting a multidrug-resistance phenotype were recovered from 45 patients. These isolates were found in blood $(26.9 \% ; 18$ of $67)$, in respiratory secretions $(14.9 \% ; 10$ of 67$)$, in urine $(55.2 \% ; 37$ of 67$)$, and at other sites $(3 \% ; 2$ of 67$)$. According to the phenotypic testing, 47 isolates were ESBL producers, including 42 Klebsiella pneumoniae, 4 Escherichia coli, and 1 Enterobacter aerogenes. The remaining 20 isolates (15 Enterobacter cloacae, $4 \mathrm{E}$. aerogenes, and $1 \mathrm{E}$. coli) were categorized as CRE because they were able to hydrolyze at least 1 carbapenem agent (ie, a single E. aerogenes was resistant to meropenem and ertapenem while others were only resistant to ertapenem). Notably, decreased susceptibility to any carbapenem agent among many (if not all) CRE isolates is related to the production of an ESBL and/or AmpC enzymes associated with membrane impermeability. In this study, the "majority mechanism" regarding the spectrum of antimicrobial hydrolysis detected by the phenotypic tests was considered.

Of the 67 isolates, 11 (16.4\%) were recovered from 9 distinct patients and were resistant to PMB: 8 ESBL-producing K. pneumoniae, 1 ESBL-producing E. coli, 1 ESBL-producing

TA BLE 1. Microbiological Features of the PMB-R MDR Noncarbapenemase-Producing Enterobacteriaceae Evaluated in This Study

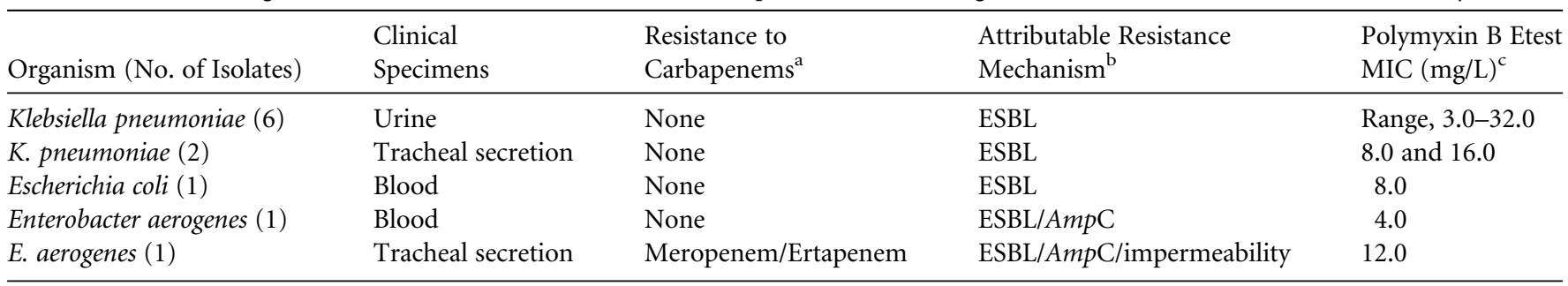

NOTE. PMB-R MDR, polymyxin B-resistant multidrug resistant; MIC, minimum inhibitory concentration; ESBL, extended spectrum

$\beta$-lactamase.

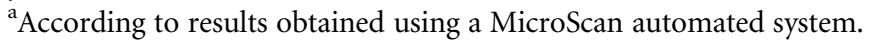

${ }^{\mathrm{b}}$ Attributable resistance mechanism for a MDR phenotype inferred by phenotypic tests.

${ }^{\mathrm{c} C o n s i d e r i n g} \leq 2 \mathrm{mg} / \mathrm{L}$ and $>2 \mathrm{mg} / \mathrm{L}$ as susceptible and resistant, respectively. 
E. aerogenes, and 1 carbapenem-resistant $E$. aerogenes. The microbiological data regarding the PMB-resistant enterobacterial species found in this study are shown in Table 1.

Resistance to carbapenems (formerly cephalosporins) among Enterobacteriaceae does matter, which could be interpreted as strong justification to be more liberal with polymyxins (formerly carbapenems) in empirical therapies. ${ }^{6}$ However, the degree to which this can be reflected among MDR organisms other than carbapenem-resistant ones, equally recovered from a setting with a high-level selection pressure, has not been evaluated properly.

Although the resistance rate was lower among MDR Enterobacteriaceae than among KPC producers $(16.4 \%$ vs $34.8 \%$, respectively) during the same period of evaluation, the outcome reported here is an important matter (and not a myth) because it illustrates a possible influence of PMB use on other bacteria whose infectious processes need not be treated with it.

Additionally, some important observations in this study follow: First, $K$. pneumoniae seems to be a specie with fitness for resistance acquisition. Second, although not as notable as the bloodstream, for example, the urinary site appears to be a reservoir for MDR organisms. Third, PMB resistance does occur without carbapenem resistance: among the $11 \mathrm{PMB}$-resistant isolates found in this study, only one E. aerogenes was a CRE (Table 1).

In conclusion, in this study, an important PMB resistance rate was detected among MDR Enterobacteriaceae isolates with the exception of the carbapenemase-producing ones. This finding emphasizes the need for a constant monitoring program to prevent the emergence of PMB resistance, not only among carbapenemase producers but especially among organisms that have a fitness to develop it.

\section{ACKNOW LEDGMENTS}

Financial support: This work was supported in part by Conselho Nacional de Desenvolvimento Científico e Tecnológico (CNPq), Brazil.

Potential conflicts of interest: The author reports no conflicts of interest relevant to this article.

\section{Leandro Reus Rodrigues Perez, $\mathrm{PhD}^{1,2}$}

Affiliations: 1. Hospital Mãe de Deus, Porto Alegre, Brazil; 2. Universidade Federal do Rio Grande do Sul, Porto Alegre, Brazil.

Address correspondence to Leandro Reus Rodrigues Perez, PhD, Microbiology Unit, Hospital Mãe de Deus, 286, José de Alencar Street, 90610-000, Porto Alegre, RS, Brazil (leandro.reus@gmail.com).

Infect Control Hosp Epidemiol 2017;38:126-127

(C) 2016 by The Society for Healthcare Epidemiology of America. All rights reserved. 0899-823X/2017/3801-0022. DOI: 10.1017/ice.2016.268

\section{REFERENCES}

1. Li J, Nation RL, Turnidge JD, et al. Colistin: the re-emerging antibiotic for multidrug-resistant gram-negative bacterial infections. Lancet Infect Dis 2006;6:589-601.

2. Gales AC, Jones RN, Sader HS. Contemporary activity of colistin and polymyxin B against a worldwide collection of gram- negative pathogens: results from the SENTRY Antimicrobial Surveillance Program (2006-09). J Antimicrob Chemother 2011; 66:2070-2074.

3. Perez LR, Dias CG. Emergence of infections due to a polymyxin B-resistant KPC-2-producing Klebsiella pneumoniae in critically ill patients: what is the role of a previous colonization. Infect Control Hosp Epidemiol 2016;37:240-241.

4. van Duin D, Doi Y. Outbreak of colistin-resistant, carbapenemase-producing Klebsiella pneumoniae: Are we at the end of the road. J Clin Microbiol 2015;53:3116-3117.

5. Perez LR. Carbapenem-resistant Enterobacteriaceae: a major prevalence difference due to the high performance of carbapenemase producers when compared to the nonproducers. Infect Control Hosp Epidemiol 2015;36:1480-1482.

6. Lee J, Patel G, Huprikar S, Calfee DP, Jenkins SG. Decreased susceptibility to polymyxin B during treatment for carbapenemresistant Klebsiella pneumoniae infection. J Clin Microbiol 2009;47:1611-1612.

\section{Fear of Failure: Engaging Patients in Antimicrobial Stewardship after Fecal Transplantation for Recurrent Clostridium difficile Infection}

To the Editor-Patients and family members are often perceived as part of the problem driving inappropriate use of antibiotics. Patients may request antibiotics due to factors such as the fear of adverse consequences if an infection is not treated or the belief that antibiotics will help them get better faster. ${ }^{1}$ In our practices, patients commonly request antibiotics for vague complaints such as fatigue that they believe must be due to a urinary tract infection (UTI) despite the absence of urinary symptoms. Prior experiences in the healthcare system usually underlie such requests: inappropriate prescription of antibiotics for self-limited conditions such as viral upper respiratory infections (URTI) or asymptomatic bacteriuria leads to the belief that antibiotics may be beneficial despite controlled trials demonstrating no benefit. ${ }^{2,3}$

The request for an antibiotic is further strengthened by a belief that antibiotics are relatively harmless; thus, the possible benefit outweighs any risk. ${ }^{3,4}$ In contrast to most patients, fecal microbiota transplantation (FMT) recipients for recurrent Clostridium difficile infection (CDI) have personal knowledge of the adverse consequences of antibiotics and are highly motivated to avoid antibiotics to prevent failure of the transplant. In our FMT practices, FMT recipients are encouraged to contact their FMT providers and/or have their physicians contact the FMT providers for consultation regarding antibiotic prescriptions after the transplant. Here, we report our experience with this antimicrobial stewardship intervention. 